\title{
Cloud Computing Alternative or the Future
}

\author{
Jiri Navratil \\ CESNET, Czech Republic \\ SVen Ubik \\ CESNET, Czech Republic \\ Pavel Pečiva \\ KZ a.s. Ústí nad Labem, Czech Republic
}

\section{Abstract}

Modern IT intensively penetrates into various fields and changing them. We could see these trends in education, in medicine and in the culture. In last decade CESNET supported many partners from these fields. CESNET was also strongly involved in traditional form of IT, which is scientific calculation. Heavy users can use faster processors and new sophisticated applications which are able to process much bigger data. In the health care, special in the hospitals are used very sophisticated Information systems collecting complex data about all patients. Part of these IS are systems PACS (Picture Archiving and Communication Systems) which process graphical data from diagnostic devices as (CT, MRI, Digital X-ray, etc.). The museums and galleries were always important part of culture segment of society. Several years ago they had nothing special only couple of PCs. In last decade they started process of digitalization of theirs collections. All these examples demonstrate that computing facilities for such applications must be very powerful. The strong IT infrastructure is expensive and one important rule must be respected. IT infrastructure must be upgraded each 5 years to keep up with development. Such changes cost lot of money. Are the universities, hospitals, museums and other institutions which are fully dependent on state budget ready to do such innovation steps? The users need IT and they expect that level of services should grow. Everybody feel that there is no way back. Is there any economic way how to cover all these IT services? Today, there are many commercial companies which create business on this. They built huge data centers (DC) which offer sufficient computing capacity and data storage on commercial base for everybody in the form of cloud services. The commercial customers understand this situation very quickly because there are several big advantages. One of them is that customers do not need any initial investments for start. Another big advantage is flexibility and scalability which allow companies to overcome period of extreme load. Despite of visible benefits the academic world still hesitates, currently, there are many clouds available on different level and sizes. CESNET is a partner in the project "GEANT cloud Platform" which offers possibility to use the most advanced clouds.

Keywords: Cloud, Data Center DC, 4K video JEL classification: L82

\section{Introduction}

Modern IT intensively penetrates into more various fields and changing them. We could see these trends in different education fields, in the medicine and also in the culture. 


\section{Education via Internet}

In last decade CESNET supported many partners from these not traditional fields. Our IT services for them were free of charge and they do not need any extra investments, just to use it or participate on common events. Despite of these facts their activities were very low because there were no strong motivations and not too much real users. Situation has changed with higher popularity of Internet which brings new online applications and external users. We believe that CESNET played important role in this progress. We organized educational courses and demos, prepared special experimental data transmissions, and actively participated on medicine conferences to show in on-line work of top specialists doing live surgeries. With partners from Music and Dance Faculty at Prague we organized on-line video sessions, remote distributed concerts and life cyber performances. If just recently many of these examples were running in experimental mode, nowadays the applications become part of normal life and the users want to have it in permanent production.

\section{The Scientific calculations}

CESNET is strongly involved also in traditional form of IT, which is scientific calculation. Also this category has significantly advanced. Heavy users as physicists, chemists or engineers can use faster processors and new sophisticated applications which are able to process much bigger data then in the past. More applications use automatically graphical post processing which gives more complex view on the results. To economize time of human specialists the requests on processing cycles can be much shorter and processing better organized. If in the past designer of new engine or engineer calculating elements of bridges were satisfied with the results achieved in period of one day, today they can have results in several minutes.

\section{The Health care}

In the health care services and especially in the hospitals and clinics the situation also dramatically improved. During last decade many of them started to run very sophisticated Information systems collecting complex data about all patients. Part of these IS are systems PACS (Picture Archiving and Communication Systems) which process graphical data from diagnostic devices as (CT, MRI, Digital X-ray, etc.). The images and videos have high and super high resolutions (4K) what create big data volumes and problems how to store them. More details in Ubik et al. (2015), and Navratil and Peciva (2018).

\section{The Culture}

The museums and galleries were always important part of culture segment pf society Couple years ago they had nothing special only couple of PCs. In last decade they started process of digitalization of theirs collections. Today, they have many different scanning devices for 3D objects of different sizes providing super high quality of 3D pictures or video. They can use these materials for demonstration of valuable collections on Internet. Also other cultural institutions and artists use video processing or tools of virtual reality in many occasions. More info in Navratil et al. $(2015,2016)$.

\section{From Mainframes to Grids}

Historically, most of IT processing was done at home (in the universities, in research institutes, industrial enterprises or in the hospitals). IT service was fully centralized. Later, everything went into distributed solutions based on PC or workstations. Every 
department or research group had own computing resources. This model worked well for a while but it generated high demands for IT specialist which had to do service for millions of PC. Besides individual PCs or workstations which had researchers or other people on their tables, still existed needs for central IT services. These services were based on the clusters of workstations. In the beginning the cluster were built just in frame of mother organizations. Later IT people started to economize this process and to integrate clusters into bigger superclusters with distributed architecture. Era of grids has born. Facilities like this are still successfully running and provide services for many users. We can demonstrate this on example of CESNET Metacentrum (MC) which is acting today as Virtual organization for whole Czech academic community. Current CESNET MC integrates 20 individual clusters from 12 participating institutions as join activity in so called NGI (National Grid Initiative). Complex system has more than 15000 CPUs running different services. The most important role is still computation service for different types of application but it provides also other type of services, as cloud services and data storage for nearly 2000 individual users. There are used more than 30 different queves for different type of jobs. In 2017 there were processed at about 2 millions of jobs of different categories with duration from several seconds to several days with total used CPU time more than 10500 years. See details in Metacentrum (2017).

\section{Budget for Infrastructure}

All this previous examples demonstrate that computing facilities for such applications must be very powerful. Complex task is not possible to run on simple desktop machines. It needs computer systems with many CPUs, enough memory and capacities for storing high volume of data and also high speed networks for communication with partners. The IT infrastructure is expensive and one important rule must be always respected.IT infrastructure must be upgraded each 5 years to keep up with development. Such changes cost lot of money. Are the universities, hospitals, museums and other institutions in these fields which are fully dependent on state budget ready to do such innovation steps? Currently, we have for academic community three IT infrastructures. CESNET is one of them. For near future our government is planning to have just one. The users need IT and they expect that level of services should grow. Everybody feel that there is no way back. Is there any economic way how to cover all these IT services?

\section{Results}

\section{Data Centres and Cloud computing}

Today, there are many commercial companies which create business on this. They built huge data centres (DC) which offer sufficient computing capacity and data storage and also advanced applications and special libraries. They offer these IT resources on commercial base for everybody in the form of cloud services. 


\section{Data Centers}

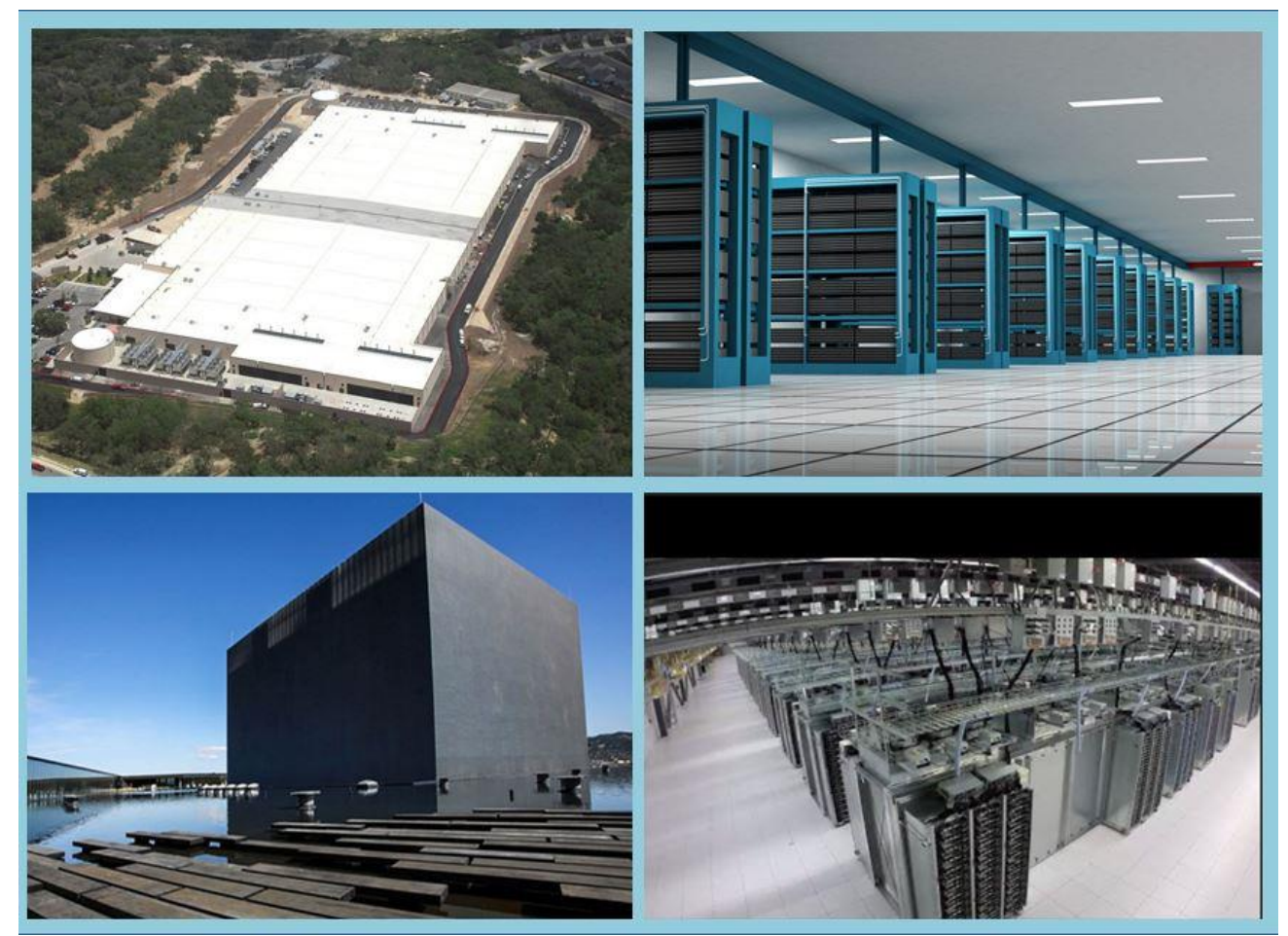

Source: Sverdlik (2012, Miller, 2017)

What is cloud service? It is form of computing where user can use offered IT resources from DC based on self-service mode. Thanks to virtualization and heavy sharing the IT resources are practically unlimited. Each cloud has own portal on which is catalogue of available services, price list for them and other important information, guides and hints, how to use it. The price is defined according demands of resources and utilized time. The user select particular service, select resources and start to use it paying for that later with VISA card, similar as in Internet on-line shops. There are several categories of cloud services.

First one is laas (Infrastructure as Service) In this case customers will use DC resources (CPUs, memory, storage, network) similar as they would buy own hardware. All software tools (operating system, databases, web etc.) will be installed and managed by own user staff. The second category is PaaS (Platform as a Service). In this case the customer use DC hardware but also use some software available in DC or use software licensed by third party. Sometimes in this model, operate Independent Software Developers, which can prepare in customer virtual environment special applications according user demands (example can be IS for hospital, On-line shop, Movie processing system, etc.) The third category of cloud services is Saas (Sofware as a Service). In that case, the customer use system running on cloud directly without any special customization. Typical example is Microsoft Office 365 running on Microsoft Azure clouds. The user uses this software via client which gives him illusion that MS Office is installed on his home computer. Categories definition are used according to the Wikipedia (2018). 
Figure 2

Category of Cloud Services and relation between partners

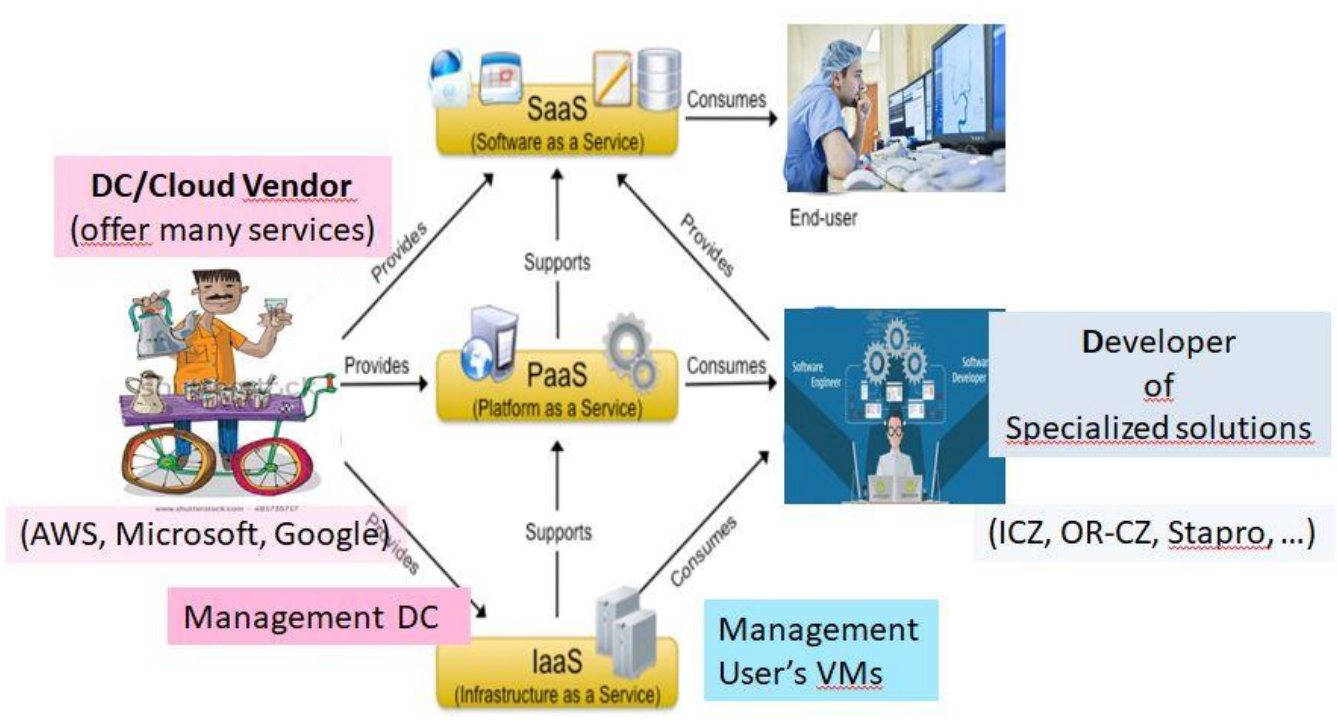

Source: Author's illustration

\section{Discussion}

In last 5 years IT world slowly moved from GRID to CLOUD. The commercial customers understand this situation very quickly. There are several big advantages. One of them is that customers do not need any initial investments to start to use IT. Another big advantage is flexibility and scalability which allow companies to overcome period of extreme load. The bills for usage are issued monthly in the mode "pay as you go". Despite of visible benefits the academic world still hesitates, they still not thinking economically the extra ordination access prevail. Fundamental questions which are open is in what moment they can leave own hardware located in their premises and to move into cloud located somewhere. It doesn't mean they have to dismiss IT staff. These specialists will be always needed but only their role will change. Sometime is overestimated question of security but according to our opinion it is very close to level which has current customer (user) on his own system.

Currently there are many clouds available on different level and sizes. CESNET is a partner in the project "GEANT cloud Platform" which offers possibility to use the most advanced clouds. In our presentation we will show interesting details from this project. The partners are Amazon with AWS (Novitz, 2017), Microsoft with Azure, CloudSigma and DimansionData with their solutions (Muspratt, 2017). On few practical demos we show how simple is to use it. See more in Geant (n.d.). We believe that academic world will soon follow this new way of using IT for its strategic and day-to-day purposes.

\section{References}

1. Navratil, J., Peciva, P. (2018), "Komercni cloud, Alternativa nebo budoucnost?" in proceedings of MEDSOFT 2018, Creative Connections, Praha, pp. 139 - 148.

2. Navratil, J., Ubik, S. (2016), "Arts and digital humanities projects a new direction of CESNET focus", in Book of abstracts s of the ENTRENOVA Conference 2016, Rovinj, pp.13-14. 
3. Navratil, J., Ubik, S., Melnikov, J. (2015) "Performing Arts Across Continents Our Way to Digital Humanities and Arts" in Proceedings of the ENTRENOVA Conference 2015, Kotor, Monte Negro, pp. 227-234.

4. Ubik, S., Navratil, J., Melnikov, J., Schraml, J., Broul, M., Peciva, P. (2015) "Internaldiscipline Collaboration in Medical Teaching", in Proceedings of the $12^{\text {th }}$ International Conference CDVE 2015, September 20-23, Mallorca, Spain, Springer, pp.220-227.

5. Metacentrum (2017), "MetaCentrum VO - virtuální organizace pro celou akademickou obec", Available at:

https://metavo.metacentrum.cz/cs/state/stats/2017/index.html/ Accessed 15th August, 2018

6. Geant (n.a.). Geant Cloud Services. Available at: https://www.geant.org/Services/Storage_and_clouds/Pages/Cloud_Services.aspx / Accessed 15 th August, 2018

7. Novitz, M. (2017). Amazon to Bring 12 More Data Centers to Ohio. Available at: https://www.transformingnetworkinfrastructure.com/topics/hyperscale-datacenters/articles/435504-amazon-bring-12-more-data-centers-ohio.htm / Accessed 15th August, 2018

8. Sverdlik, Y. (2012). Microsoft adds new data centers to azure infrastructure. Available at:

http://archive.datacenterdynamics.com/focus/archive/2012/04/microsoft-adds-newdata-centers-azure-infrastructure / Accessed 15th August, 2018

9. Miller, R. (2017). Facebook Comes to Richmond, Boosting Green Energy Options in Virginia. Available at: https://datacenterfrontier.com/facebook-comes-to-richmond-boosting-greenenergy-options-in-virginia/ / Accessed 15th August, 2018

10. Wikipedia (2018). Cloud computing. https://en.wikipedia.org/wiki/Cloud_computing/ Accessed 15th August, 2018

11. Muspratt, A. (2017). Born To Be Cloud. Available at: https://blog.crozdesk.com/tapping-saas-paas-iaas/ / Accessed 15th August, 2018

\section{About the authors}

Jiri Navratil received his PhD in Computer Science from Czech Technical University at Prague in 1984. He worked for 30 years at Computing and Information Center of CTU in different positions linked with High Performance Computing a Communications. Since 2006 he started work for CESNET - Czech Education a Scientific Network as leader of group supporting special research applications. The author can be contacted at jiri@cesnet.cz.

Sven Ubik is senior researcher in CESNET. He received his MSc. and Dr. in Computer Science from the Czech Technical University in 1990 and 1998, respectively. His research interests include network monitoring, high-definition video, programmable hardware and optical networks. The author can be contacted at ubik@cesnet.cz.

Pavel Pečiva is IT specialists in the hospital which organize many on-line events. He is senior technician responsible for production of video sessions and video and sound editor. The author can be contacted at pavel.peciva@kzcr.eu. 